PROCEEDINGS OF THE

AMERICAN MATHEMATICAL SOCIETY

Volume 138, Number 7, July 2010, Pages 2375-2379

S 0002-9939(10)10324-4

Article electronically published on March 15, 2010

\title{
A NOTE ON VALUES OF NONCOMMUTATIVE POLYNOMIALS
}

\author{
MATEJ BREŠAR AND IGOR KLEP
}

(Communicated by Nigel J. Kalton)

\begin{abstract}
We find a class of algebras $\mathcal{A}$ satisfying the following property: for every nontrivial noncommutative polynomial $f\left(X_{1}, \ldots, X_{n}\right)$, the linear span of all its values $f\left(a_{1}, \ldots, a_{n}\right), a_{i} \in \mathcal{A}$, equals $\mathcal{A}$. This class includes the algebras of all bounded and all compact operators on an infinite dimensional Hilbert space.
\end{abstract}

\section{INTRODUCTION}

Starting with Helton's seminal paper Hel there has been considerable interest over the last years in values of noncommuting polynomials on matrix algebras. In one of the papers in this area the second author and Schweighofer [KS] showed that Connes' embedding conjecture is equivalent to a certain algebraic assertion which involves the trace of polynomial values on matrices. This has motivated us [BK] to consider the linear span of values of a noncommutative polynomial $f$ on the matrix algebra $M_{d}(\mathbb{F})$; here, $\mathbb{F}$ is a field with $\operatorname{char}(\mathbb{F})=0$. It turns out [BK, Theorem 4.5] that this span can be either:

(1) $\{0\}$;

(2) the set of all scalar matrices;

(3) the set of all trace zero matrices; or

(4) the whole algebra $M_{d}(\mathbb{F})$.

From the precise statement of this theorem it also follows that if $2 d>\operatorname{deg} f$, then (1) and (2) do not occur and (3) occurs only when $f$ is a sum of commutators.

What to except in infinite dimensional analogues of $M_{d}(\mathbb{F})$ ? More specifically, let $\mathcal{H}$ be an infinite dimensional Hilbert space, and let $\mathcal{B}(\mathcal{H})$ and $\mathcal{K}(\mathcal{H})$ denote the algebras of all bounded and compact linear operators on $\mathcal{H}$, respectively. What is the linear span of polynomial values in $\mathcal{B}(\mathcal{H})$ and $\mathcal{K}(\mathcal{H})$ ? A very special (but decisive, as we shall see) case of this question was settled by Halmos [Hal and Pearcy and Topping $[\mathrm{PT}$ (see also Anderson And) a long time ago: every operator in $\mathcal{B}(\mathcal{H})$ and $\mathcal{K}(\mathcal{H})$, respectively, is a sum of commutators. That is, the linear span of values of the polynomial $X_{1} X_{2}-X_{2} X_{1}$ on $\mathcal{B}(\mathcal{H})$ and $\mathcal{K}(\mathcal{H})$ is all of $\mathcal{B}(\mathcal{H})$ and $\mathcal{K}(\mathcal{H})$, respectively. We will prove that the same is true for every nonconstant

Received by the editors September 30, 2009, and, in revised form, December 2, 2009.

2010 Mathematics Subject Classification. Primary 08B20, 16R99, 47L30.

Key words and phrases. Noncommutative polynomial, Lie ideal, Hilbert space, bounded operator, compact operator.

The first author was supported by the Slovenian Research Agency (program No. P1-0288)

The second author was supported by the Slovenian Research Agency (program No. P1-0222).

(C)2010 American Mathematical Society Reverts to public domain 28 years from publication 
polynomial. This result will be derived as a corollary of our main theorem which presents a class of algebras with the property that the span of values of "almost" every polynomial is equal to the whole algebra.

\section{Results}

By $\mathbb{F}\langle\bar{X}\rangle$ we denote the free algebra over a field $\mathbb{F}$ generated by $\bar{X}=\left\{X_{1}, X_{2}, \ldots\right\}$, i.e., the algebra of all noncommutative polynomials in $X_{i}$. Let $f=f\left(X_{1}, \ldots, X_{n}\right) \in$ $\mathbb{F}\langle\bar{X}\rangle$. We say that $f$ is homogeneous in the variable $X_{i}$ if all monomials of $f$ have the same degree in $X_{i}$. If this degree is 1 , then we say that $f$ is linear in $X_{1}$. If $f$ is linear in every variable $X_{i}, 1 \leq i \leq n$, then we say that $f$ is multilinear.

Let $\mathcal{A}$ be an algebra over $\mathbb{F}$. By $f(\mathcal{A})$ we denote the set of all values $f\left(a_{1}, \ldots, a_{n}\right)$ with $a_{i} \in \mathcal{A}, i=1, \ldots, n$. Recall that $f=f\left(X_{1}, \ldots, X_{n}\right) \in \mathbb{F}\langle\bar{X}\rangle$ is said to be an identity of $\mathcal{A}$ if $f(\mathcal{A})=\{0\}$. If $f(\mathcal{A})$ is contained in the center of $\mathcal{A}$, but $f$ is not an identity of $\mathcal{A}$, then $f$ is said to be a central polynomial of $\mathcal{A}$. By $\operatorname{span} f(\mathcal{A})$ we denote the linear span of $f(\mathcal{A})$. We are interested in the question, when does $\operatorname{span} f(\mathcal{A})=\mathcal{A}$ hold?

For the proof of our main theorem three rather elementary lemmas will be needed. The first and the simplest one is a slightly simplified version of BK, Lemma 2.2]. Its proof is based on the standard Vandermonde argument.

Lemma 2.1. Let $\mathcal{V}$ be a vector space over an infinite field $\mathbb{F}$, and let $\mathcal{U}$ be a subspace. Suppose that $c_{0}, c_{1}, \ldots, c_{n} \in \mathcal{V}$ are such that $\sum_{i=0}^{n} \lambda^{i} c_{i} \in \mathcal{U}$ for all $\lambda \in \mathbb{F}$. Then each $c_{i} \in \mathcal{U}$.

Recall that a vector subspace $\mathcal{L}$ of $\mathcal{A}$ is said to be a Lie ideal of $\mathcal{A}$ if $[\ell, a] \in \mathcal{L}$ for all $\ell \in \mathcal{L}$ and $a \in \mathcal{A}$; here, $[u, v]=u v-v u$. For a recent treatise on Lie ideals from an algebraic as well as functional analytic viewpoint we refer the reader to BKS.

Our second lemma is a special case of [BK], Theorem 2.3].

Lemma 2.2. Let $\mathcal{A}$ be an algebra over an infinite field $\mathbb{F}$, and let $f \in \mathbb{F}\langle\bar{X}\rangle$. Then $\operatorname{span} f(\mathcal{A})$ is a Lie ideal of $\mathcal{A}$.

Every vector subspace of the center of $\mathcal{A}$ is obviously a Lie ideal of $\mathcal{A}$. Lie ideals that are not contained in the center are called noncentral. The third lemma follows from an old result of Herstein [Her, Theorem 1.2].

Lemma 2.3. Let $\mathcal{S}$ be a simple algebra over a field $\mathbb{F}$ with $\operatorname{char}(\mathbb{F}) \neq 2$. If $\mathcal{M}$ is both a noncentral Lie ideal of $\mathcal{S}$ and a subalgebra of $\mathcal{S}$, then $\mathcal{M}=\mathcal{S}$.

We are now in a position to prove our main result.

Theorem 2.4. Let $\mathcal{S}$ and $\mathcal{B}$ be algebras over a field $\mathbb{F}$ with $\operatorname{char}(\mathbb{F})=0$, and let $\mathcal{A}=\mathcal{S} \otimes \mathcal{B}$. Suppose that $\mathcal{S}$ is simple, and suppose that $\mathcal{B}$ satisfies

(a) every element in $\mathcal{B}$ is a sum of commutators; and

(b) for each $n \geq 1$, every element in $\mathcal{B}$ is a linear combination of elements $b^{n}$, $b \in \mathcal{B}$.

If $f \in \mathbb{F}\langle\bar{X}\rangle$ is neither an identity nor a central polynomial of $\mathcal{S}$, then

$$
\operatorname{span} f(\mathcal{A})=\mathcal{A} \text {. }
$$

(In case $\mathcal{A}$ is nonunital, only polynomials $f$ with zero constant term are considered.) 
Proof. Let $f=f\left(X_{1}, \ldots, X_{n}\right)$. Let us write $f=g_{i}+h_{i}$ where $g_{i}$ is a sum of all monomials of $f$ in which $X_{i}$ appears and $h_{i}$ is a sum of all monomials of $f$ in which $X_{i}$ does not appear. Thus, $h_{i}=h_{i}\left(X_{1}, \ldots, X_{i-1}, X_{i+1}, \ldots, X_{n}\right)$ and hence

$$
h_{i}\left(a_{1}, \ldots, a_{i-1}, a_{i+1}, \ldots, a_{n}\right)=f\left(a_{1}, \ldots, a_{i-1}, 0, a_{i+1}, \ldots, a_{n}\right)
$$

for all $a_{i} \in \mathcal{A}$. Therefore $\operatorname{span} h_{i}(\mathcal{A}) \subseteq \operatorname{span} f(\mathcal{A})$, which clearly implies $\operatorname{span} g_{i}(\mathcal{A})$ $\subseteq$ span $f(\mathcal{A})$. At least one of $g_{i}$ and $h_{i}$ is neither an identity nor a central polynomial of $\mathcal{S}$. Therefore there is no loss of generality in assuming that either $X_{i}$ appears in every monomial of $f$ or $f$ does not involve $X_{i}$ at all. Since $f$ cannot be a constant polynomial and hence it must involve some of the $X_{i}$ 's, we may assume, again without loss of generality, that each monomial of $f$ involves all $X_{i}, i=1, \ldots, n$.

Next we claim that there is no loss of generality in assuming that $f$ is homogeneous in $X_{1}$. Write $f=f_{1}+\ldots+f_{m}$, where $f_{i}$ is the sum of all monomials of $f$ that have degree $i$ in $X_{1}$. Note that

$$
f\left(\lambda a_{1}, a_{2}, \ldots, a_{n}\right)=\sum_{i=1}^{m} \lambda^{i} f_{i}\left(a_{1}, \ldots, a_{n}\right) \in \operatorname{span} f(\mathcal{A})
$$

for all $\lambda \in \mathbb{F}$ and all $a_{i} \in \mathcal{A}$, so $f_{i}\left(a_{1}, \ldots, a_{n}\right) \in \operatorname{span} f(\mathcal{A})$ by Lemma 2.1. Thus, $\operatorname{span} f_{i}(\mathcal{A}) \subseteq \operatorname{span} f(\mathcal{A})$. At least one $f_{i}$ is neither an identity nor a central polynomial of $\mathcal{S}$. Therefore it suffices to prove the theorem for $f_{i}$. This proves our claim.

Let us now show that there is no loss of generality in assuming that $f$ is linear in $X_{1}$. If $\operatorname{deg}_{X_{1}} f>1$, we apply the multilinearization process to $f$; i.e., we introduce a new polynomial $\Delta_{1, n+1} f=f^{\prime}\left(X_{1}, \ldots, X_{n}, X_{n+1}\right)$ :

$$
f^{\prime}=f\left(X_{1}+X_{n+1}, X_{2}, \ldots, X_{n}\right)-f\left(X_{1}, X_{2}, \ldots, X_{n}\right)-f\left(X_{n+1}, X_{2}, \ldots, X_{n}\right) .
$$

This reduces the degree in $X_{1}$ by one. Clearly, span $f^{\prime}(\mathcal{A}) \subseteq \operatorname{span} f(\mathcal{A})$. Observe that $f$ can be retrieved from $f^{\prime}$ by resubstituting $X_{n+1} \mapsto X_{1}$; more exactly

$$
\left(2^{\operatorname{deg}_{X_{1}} f}-2\right) f=f^{\prime}\left(X_{1}, \ldots, X_{n}, X_{1}\right) .
$$

Hence $f^{\prime}$ is not an identity nor a central polynomial of $\mathcal{S}$. Note however that $f^{\prime}$ is not necessarily homogeneous in $X_{1}$, but for all its homogeneous components $f_{i}^{\prime}$ we have span $f_{i}^{\prime}(\mathcal{A}) \subseteq \operatorname{span} f^{\prime}(\mathcal{A})$; one can check this by using Lemma 2.1, as in the previous paragraph. At least one of these components, say $f_{j}^{\prime}$, is not an identity nor a central polynomial of $\mathcal{S}$. Thus we restrict our attention to $f_{j}^{\prime}$. If necessary, we continue applying $\Delta_{1,-}$, and after a finite number of steps we obtain a polynomial $\Delta f$ that is linear in $X_{1}$ which is neither an identity nor a central polynomial of $\mathcal{S}$ and which satisfies $\operatorname{span} \Delta f(\mathcal{A}) \subseteq \operatorname{span} f(\mathcal{A})$. Hence we may assume $f$ is linear in $X_{1}$.

Repeating the same argument with respect to other variables we finally see that without loss of generality we may assume that $f$ is multilinear.

Set $\mathcal{L}=\operatorname{span} f(\mathcal{A})$ and $\mathcal{M}=\{m \in \mathcal{S} \mid m \otimes \mathcal{B} \subseteq \mathcal{L}\}$. By Lemma 2.2, $\mathcal{L}$ is a Lie ideal of $\mathcal{A}$. Therefore $[m, s] \otimes b^{2}=[m \otimes b, s \otimes b] \in \mathcal{L}$ for all $m \in \mathcal{M}, b \in \mathcal{B}$, $s \in \mathcal{S}$. Using (b) it follows that $[m, s] \in \mathcal{M}$. Therefore $\mathcal{M}$ is a Lie ideal of $\mathcal{S}$. Pick $s_{1}, \ldots, s_{n} \in \mathcal{S}$ such that $s_{0}=f\left(s_{1}, \ldots, s_{n}\right)$ does not lie in the center of $\mathcal{S}$. For every $b \in \mathcal{B}$ we have

$$
s_{0} \otimes b^{n}=f\left(s_{1} \otimes b, s_{2} \otimes b, \ldots, s_{n} \otimes b\right) \in \mathcal{L} .
$$


In view of (b) this yields $s_{0} \in \mathcal{M}$. Accordingly, $\mathcal{M}$ is a noncentral Lie ideal of $\mathcal{S}$. Next, given $m \in \mathcal{M}$ and $b, b^{\prime} \in \mathcal{B}$, we have

$$
m^{2} \otimes\left[b, b^{\prime}\right]=\left[m \otimes b, m \otimes b^{\prime}\right] \in \mathcal{L} .
$$

By (a), this gives $m^{2} \in \mathcal{M}$. From

$$
m_{1} m_{2}=\frac{1}{2}\left(\left[m_{1}, m_{2}\right]+\left(m_{1}+m_{2}\right)^{2}-m_{1}^{2}-m_{2}^{2}\right)
$$

it now follows that $\mathcal{M}$ is a subalgebra of $\mathcal{S}$. Using Lemma 2.3 we now conclude that $\mathcal{M}=\mathcal{S}$; i.e., $\mathcal{A}=\mathcal{S} \otimes \mathcal{B} \subseteq \mathcal{L} \subseteq \mathcal{A}$.

From the identity

$$
n ! b=\sum_{i=0}^{n-1}(-1)^{n-1-i}\left(\begin{array}{c}
n-1 \\
i
\end{array}\right)\left((b+i)^{n}-i^{n}\right)
$$

it is immediate that (b) is fulfilled if $\mathcal{B}$ has a unity. In this case the proof can be actually slightly simplified by avoiding use of powers of elements in $\mathcal{B}$. Further, every $C^{*}$-algebra $\mathcal{B}$ satisfies (b). Indeed, every element in $\mathcal{B}$ is a linear combination of positive elements, and for positive elements we can define $n$th roots.

Corollary 2.5. Let $\mathcal{H}$ be an infinite dimensional Hilbert space. Then

$$
\operatorname{span} f(\mathcal{B}(\mathcal{H}))=\mathcal{B}(\mathcal{H})
$$

for every nonconstant polynomial $f \in \mathbb{C}\langle\bar{X}\rangle$.

Proof. It is well known that there does not exist a nonzero polynomial that is an identity of $M_{d}(\mathbb{C})$ for every $d \geq 1$, cf. [Row, Lemma 1.4.3]. Therefore there exists $d \geq 1$ such that $\left[f, X_{n+1}\right]$ is not an identity of $M_{d}(\mathbb{C})$. This means that $f$ is neither an identity nor a central polynomial of $M_{d}(\mathbb{C})$. Since $\mathcal{H}$ is infinite dimensional, we have $\mathcal{B}(\mathcal{H}) \cong M_{d}(\mathcal{B}(\mathcal{H})) \cong M_{d}(\mathbb{C}) \otimes \mathcal{B}(\mathcal{H})$. Now we are in a position to use Theorem 2.4 Indeed, $M_{d}(\mathbb{C})$ is a simple algebra, and the algebra $\mathcal{B}(\mathcal{H})$ satisfies (a) by [Hal, and satisfies (b) since it is unital.

Corollary 2.6. Let $\mathcal{H}$ be an infinite dimensional Hilbert space. Then

$$
\operatorname{span} f(\mathcal{K}(\mathcal{H}))=\mathcal{K}(\mathcal{H})
$$

for every nonzero polynomial $f \in \mathbb{C}\langle\bar{X}\rangle$ with zero constant term.

Proof. The proof is essentially the same as that of Corollary 2.5. The only difference occurs in verifying whether $\mathcal{K}(\mathcal{H})$ satisfies the conditions of Theorem 2.4, For this we just note that (a) is shown in $[\mathrm{PT}]$, and (b) follows by the remark preceding the statement of Corollary 2.5 .

\section{REFERENCES}

[And] J. Anderson, Commutators of compact operators, J. Reine Angew. Math. 291 (1977) 128-132. MR0442742 (56:1122)

[BKS] M. Brešar, E. Kissin, V. Shulman, Lie ideals: from pure algebra to $C^{*}$-algebras, J. Reine Angew. Math. 623 (2008) 73-121. MR2458041 (2009i:47168)

[BK] M. Brešar, I. Klep, Values of noncommutative polynomials, Lie skew-ideals and tracial Nullstellensätze, Math. Res. Lett. 16 (2009) 605-626. MR2525028

[Hal] P. R. Halmos, Commutators of operators II, Amer. J. Math. 76 (1954) 191-198. MR 0059484(15:538d)

[Hel] J. W. Helton, "Positive" noncommutative polynomials are sums of squares, Ann. of Math. (2) 156 (2002) 675-694. MR1933721 (2003k:12002) 
[Her] I. N. Herstein, Topics in ring theory, The University of Chicago Press, 1969. MR.0271135(42:6018)

[KS] I. Klep, M. Schweighofer, Connes' embedding conjecture and sums of Hermitian squares, Adv. Math. 217 (2008) 1816-1837. MR.2382741 (2009g:46109)

[PT] C. Pearcy, D. Topping, On commutators in ideals of compact operators, Michigan J. Math. 18 (1971) 247-252. MR0284853 (44:2077)

[Row] L. H. Rowen, Polynomial identities in ring theory, Academic Press, 1980. MR.576061 (82a:16021)

Faculty of Mathematics and Physics, University of Ljubljana, Jadranska Ulica 19, SI-1000 Ljubluana, Slovenia - And - Faculty of Natural Sciences and Mathematics, University of Maribor, KorošKa 160, SI-2000 Maribor, Slovenia

E-mail address: matej.bresar@fmf.uni-lj.si

Faculty of Mathematics and Physics, University of Ljubljana, Jadranska ulica 19, SI-1000 Ljubljana, Slovenia - And - Faculty of Natural Sciences and Mathematics, University of Maribor, Koroška 160, SI-2000 Maribor, Slovenia

E-mail address: igor.klep@fmf.uni-lj.si 